\title{
Simplicial volume and fillings of hyperbolic manifolds
}

\author{
KOJI FUJIWARA \\ JASON FOX MANNING
}

Let $M$ be a hyperbolic $n$-manifold whose cusps have torus cross-sections. In [8], the authors constructed a variety of nonpositively and negatively curved spaces as " $2 \pi$-fillings" of $M$ by replacing the cusps of $M$ with compact "partial cones" of their boundaries. These $2 \pi$-fillings are closed pseudomanifolds, and so have a fundamental class. We show that the simplicial volume of any such $2 \pi-$ filling is positive, and bounded above by $\frac{\operatorname{Vol}(M)}{v_{n}}$, where $v_{n}$ is the volume of a regular ideal hyperbolic $n$-simplex. This result generalizes the fact that hyperbolic Dehn filling of a 3-manifold does not increase hyperbolic volume.

In particular, we obtain information about the simplicial volumes of some 4-dimensional homology spheres described by Ratcliffe and Tschantz, answering a question of Belegradek and establishing the existence of 4-dimensional homology spheres with positive simplicial volume.

20F65, 53C23

\section{Introduction}

Simplicial volume was defined for manifolds by Gromov in [11]. For an $n$-dimensional closed manifold $M$ of constant sectional curvature $K=-1$, the simplicial volume $\|M\|$ is proportional to the (Riemannian) volume $\operatorname{Vol}(M)$, and the ratio is the volume of a regular ideal hyperbolic $n$-simplex; in particular, it depends only on the dimension [11, $0.4]$. More generally (see [11, 0.3, 0.5]) for constants $a \leq b<0$, there is a constant $C=C(a, b, n)>1$ so that if the sectional curvatures $K$ satisfy $a \leq K \leq b$, then

$$
\operatorname{Vol}(M) / C \leq\|M\| \leq C \operatorname{Vol}(M) .
$$

This note is motivated in part by the (apparently open) question:

Question 1.1 Fix $v>0$, and let $\mathcal{M}_{n, v}$ be the set of (homotopy classes of) closed $n$-manifolds whose sectional curvatures are all negative, and whose simplicial volume is bounded above by $v$. Is $\mathcal{M}_{n, v}$ finite whenever $n \geq 4$ ?

Some comments, fixing $n \geq 4$ and $v>0$ : 
- The set $\mathcal{M}_{n, v}$ contains only finitely many constant curvature manifolds, even up to diffeomorphism. This follows from Wang's finiteness theorem [30] together with the proportionality of simplicial volume to hyperbolic volume for closed manifolds (see, for example, Benedetti and Petronio [4, C.4]).

- Fix $b>0$. The set $\mathcal{M}_{n, v}$ contains only finitely many diffeomorphism classes of manifolds with sectional curvatures $K$ pinched between -1 and $b$. Indeed, as noted in the inequality (1), the curvature assumption $-1 \leq K \leq b<0$ together with an upper bound on the simplicial volume gives an upper bound on the volume (see [11, 0.3, 1.2]). On the other hand, the condition $-1 \leq K<0$ together with the upper bound on the volume gives an upper bound on the diameter (see Gromov [10, 1.2]) and a lower bound on the volume [10, 1.3 A]. Now, Cheeger's finiteness theorem applies [10, 1.4].

Thus if there are infinitely many diffeomorphism types of closed $n$-manifolds of bounded volume, with sectional curvatures $\leq-1$, there cannot be any uniform lower bound on their sectional curvatures, so long as $n \geq 4$. So a negative answer to Question 1.1 would have to involve manifolds without uniformly pinched curvature. For $n>3$ there do exist sequences of negatively curved manifolds whose curvatures cannot be uniformly pinched. Gromov and Thurston [13, 0.5] describe a sequence $\left\{V_{i}\right\}$ of negatively curved closed manifolds which are $i$-fold ramified covers of a fixed closed manifold $V$ of constant negative curvature, and show the curvatures of the $V_{i}$ cannot be uniformly pinched. We remark that such a sequence of ramified covers $V_{i}$ must satisfy $0<i\|V\| \leq\left\|V_{i}\right\|$, so it does not imply a negative answer to Question 1.1.

- The sets $\mathcal{M}_{3, v}$ are infinite for large enough $v$, even if one restricts to constant curvature. This is because of Thurston's hyperbolic Dehn surgery theorem, and Thurston's theorem (which we generalize in this note) that volume decreases under hyperbolic Dehn filling.

It is easy to extend the definition of simplicial volume to closed pseudomanifolds. This is explained in Section 2 (see also [31]). Pseudomanifold versions of the bounds in (1) have been established by Yamaguchi [31, Theorem 0.2 and 0.5 ] as follows. First, if $X$ is an orientable $n$-dimensional, compact, geodesically complete (locally) CAT $(-1)$ pseudo-manifold, then

$$
\operatorname{Vol}(X) \leq \frac{\pi}{(n-1) !}\|X\|
$$

Yamaguchi conjectures that the factor of $\frac{\pi}{(n-1) !}$ in (2) can be replaced by the volume of a regular ideal hyperbolic $n$-simplex. Second, if $X$ is an $n$-dimensional compact orientable geodesic space without boundary such that $K \geq-1$ in the sense of Alexandrov 
and $X$ is "locally Lipschitz contractible", then

$$
\|X\| \leq n !(n-1)^{n} \operatorname{Vol}(X),
$$

where $\operatorname{Vol}(X)$ is the $n$-dimensional Hausdorff measure of $X$. Both bounds cannot be applied at once except in the manifold setting, since upper and lower curvature bounds can only coexist in a manifold. We do not use either bound in this paper, except in Remark 1.11 below.

We do not answer Question 1.1, but we answer the analogous question for negatively curved pseudomanifolds:

Question 1.2 Let $n \geq 4$. Fix $v>0$, and let $\mathcal{P}_{n, v}$ be the set of (homotopy classes of) closed locally CAT(-1) $n$-pseudomanifolds whose simplicial volume is bounded above by $v$. Is $\mathcal{P}_{n, v}$ finite?

Specifically, we apply a "Dehn filling" construction to give a negative answer to Question 1.2.

Let $M$ be a compact $n$-manifold with boundary a union of $m$ tori, whose interior $V=\operatorname{Int}(M)$ admits a complete hyperbolic metric of finite volume. Let $\bar{M} \cong M$ be a compact manifold obtained from $V$ by removing horospherical neighborhoods of the cusps. The manifold $\bar{M}$ has $m$ boundary components $N_{1}, \ldots, N_{m}$, each of which inherits a flat metric from the hyperbolic metric on $\operatorname{Int}(M)$. In [8], we defined and studied fillings of $M$, which are topological spaces obtained by gluing the product of a torus with the cone on another torus to each boundary component of $\bar{M}$. Here is an equivalent definition.

Definition 1.3 For each $i \in\{1, \ldots, m\}$, let $T_{i}<N_{i}$ be a $k_{i}$-dimensional submanifold which is totally geodesic in $N_{i}$. The $(n-1)$-torus $N_{i}$ is foliated by parallel copies of $T_{i}$, with leaf space $V_{i}$ homeomorphic to an $\left(n-1-k_{i}\right)$-dimensional torus. Let $h_{i}: N_{i} \rightarrow V_{i}$ be the quotient map, and define $M\left(T_{1}, \ldots, T_{m}\right)$ to be the quotient $\bar{M} / \sim$, where $x \sim x^{\prime}$ if $h_{i}(x)=h_{i}\left(x^{\prime}\right)$ for some $i$. The space $M\left(T_{1}, \ldots, T_{m}\right)$ is called a filling of $M$. If all of the tori $T_{i}$ have injectivity radius strictly larger than $\pi$, $M\left(T_{1}, \ldots, T_{m}\right)$ is called a $2 \pi$-filling of $M$.

In case $M$ is 3-dimensional, and each $T_{i}$ is one-dimensional, fillings $M\left(T_{1}, \ldots, T_{m}\right)$ are the same as ordinary Dehn fillings of $M$. In [8], we generalize work of Bleiler and Hodgson [5], Schroeder [28] and Mosher and Sageev [23] (including the GromovThurston $2 \pi$ Theorem) to prove: 
Theorem 1.4 (Fujiwara and Manning [8]) Let $M$ be an $n$-dimensional hyperbolic manifold as above. Every $2 \pi$-filling $M\left(T_{1}, \ldots, T_{m}\right)$ of $M$ admits a locally $C A T(0)$ metric, so the universal cover is $C A T(0)$ with isolated flats. If every $T_{i}$ has dimension at least $n-2$, then $M\left(T_{1}, \ldots, T_{m}\right)$ admits a locally $C A T(-1)$ metric.

Each filling of $M$ is a closed pseudomanifold with singular set equal to a disjoint union of tori of dimensions $n-\left(1+\operatorname{dim}\left(T_{1}\right)\right), \ldots n-\left(1+\operatorname{dim}\left(T_{m}\right)\right)$ (see Definition 2.1 for these terms). In particular, its simplicial volume is well-defined (see Yamaguchi [31]). The main result of the current paper is that these fillings have positive simplicial volume, bounded above by the simplicial volume of $M$.

Theorem 1.5 Let $M$ be a compact $n$-manifold with boundary a union of (n-1)-tori, and suppose that $V=\operatorname{Int}(M)$ admits a complete hyperbolic metric of finite volume $\operatorname{Vol}(V)$. Let $M\left(T_{1}, \ldots, T_{m}\right)$ be a $2 \pi$-filling of $\operatorname{Int}(M)$. Then

$$
0<\left\|M\left(T_{1}, \ldots, T_{m}\right)\right\| \leq \frac{\operatorname{Vol}(V)}{v_{n}},
$$

where $v_{n}$ is the volume of a regular ideal hyperbolic $n$-simplex.

Corollary 1.6 The answer to Question 1.2 is "no," for $v$ equal to the volume of any hyperbolic $n$-manifold with torus cusps.

Proof Let $M, \bar{M}, N_{1}, \ldots, N_{m}$ be as in the discussion preceding Definition 1.3. For each $j \in\{1, \ldots, m\}$, choose a sequence $\left\{T_{i}^{j}\right\}_{i \in \mathbb{N}}$ of totally geodesic codimension 1 tori in $N_{j}$, all with inj $>\pi$, and so that $\lim _{i \rightarrow \infty} \operatorname{inj}\left(T_{i}^{j}\right)=\infty$. Let $M_{i}$ be the filling $M\left(T_{i}^{1}, \ldots, T_{i}^{m}\right)$. [8, Proposition 2.12] implies that for any $i$ only finitely many $M_{j}$ have fundamental group isomorphic to $\pi_{1} M_{i}$, so the set $\left\{M_{i}\right\}_{i \in \mathbb{N}}$ contains infinitely many homotopy classes. Theorem 1.4 implies that $0<\left\|M_{i}\right\| \leq \frac{\operatorname{Vol}(V)}{v_{n}}$ for all $i$.

Remark 1.7 In [27], Ratcliffe and Tschantz describe some nonpositively curved 4dimensional homology spheres. These homology spheres are obtained by $2 \pi$-filling of a cusped hyperbolic manifold described by Ivanšić in [18]. They seem to be the first exmples of 4-dimensional homology spheres with Riemannian metrics of non-positive sectional curvature (see Nabutovsky and Weinberger [24, paragraph after Remark 5]). Belegradek in [3] asked if these homology spheres have positive simplicial volume. Our main theorem answers this question affirmatively, but the main part of our argument is to do with the upper bound on simplicial volume. The reader primarily interested in positivity of simplicial volume can stop with Section 2.1. In particular, we answer Belegradek's question with Corollary 2.5 at the end of that subsection. 
It would be interesting to get some further information on the possible volumes of fillings of $M$. Hyperbolic Dehn filling of a 3-manifold strictly decreases volume, and the volume difference goes to zero as the length of the filling slope goes to infinity (see Thurston [29, 6.5.6] for strictness and Neumann-Zagier [25], Hodgson-Kerckhoff [16] and Futer-Kalfagianni-Purcell [9] for estimates of the difference). We conjecture that similar phenomena hold in higher dimensions.

Conjecture 1.8 The volumes $\left\|M\left(T_{1}, \ldots, T_{m}\right)\right\|$ accumulate on $\frac{\operatorname{Vol}(V)}{v_{n}}$ but do not attain it.

Question 1.9 Let $\epsilon>0$. Is there some $R$ so that if every torus $T_{i}$ has injectivity radius bigger than $R$, then

$$
\frac{\operatorname{Vol}(V)}{v_{n}}-\epsilon<\left\|M\left(T_{1}, \ldots, T_{m}\right)\right\|<\frac{\operatorname{Vol}(V)}{v_{n}} ?
$$

Question 1.10 Consider the set of fundamental groups of $2 \pi$-fillings of $M$ as a subspace of the space of "marked groups" generated by some fixed set of generators of $\pi_{1} M$. Is the simplicial volume a continuous function on this space?

Remark 1.11 It is possible to analyze the metrics we constructed in [8] and apply Yamaguchi's inequality (2) to deduce that the CAT(-1) fillings of a fixed $M$ have simplicial volume bounded away from zero, but this is about all we know for certain.

\subsection{Outline}

In Section 2.1 we recall the definition of simplicial volume for manifolds and pseudomanifolds, and apply work of Mineyev-Yaman to obtain the lower bound in the main theorem. Section 2.2 and Appendix B are technical sections concerned with replacing singular chains by "symmetric" singular chains. The upper bound of the main theorem is proved in Section 3. In Appendix A we give a proof of the proportionality of volume and relative simplicial volume for cusped hyperbolic manifolds.

\section{Simplicial volume and duality}

In this section we recall the definition of simplicial volume for pseudomanifolds and set up some computational tools. 


\subsection{Gromov norms}

Let $X$ be any space, and let $\omega \in H_{*}(X ; \mathbb{R})$ or $\omega \in H_{*}(X, Y ; \mathbb{R})$ for some $Y \subset X$. The Gromov norm of $\omega$ is the smallest $l^{1}$-norm of a real singular chain representing $\omega$,

$$
\|\omega\|=\inf \left\{\sum_{i=1}^{k}\left|\alpha_{i}\right| \mid\left[\sum_{i=1}^{k} \alpha_{i} \sigma_{i}\right]=\omega\right\} .
$$

(Since this infimum may be zero, the "Gromov norm" is only a seminorm in general.) This quantity only depends on $\omega$ and the homotopy type of the pair $(X, Y)$. In the absolute case, we can use it to define the Gromov norm of any $\omega \in H_{*}(G)$, where $G$ is a discrete group, by choosing $X$ to be a $K(G, 1)$.

If $\omega$ is an integral cycle and $\eta$ is the canonical map from integral to real homology, then we set $\|\omega\|=\|\eta(\omega)\|$.

The Gromov norm of a cycle can be computed via the pairing between bounded cohomology and $l^{1}$ homology: If $\omega \in H_{k}(X)$ and $H_{b}^{k}(X ; \mathbb{R})$ is the $k$-dimensional bounded cohomology of $X$, then

$$
\|\omega\|=\sup \left(\left\{\frac{1}{\|\phi\|_{\infty}} \mid \phi \in H_{b}^{k}(X ; \mathbb{R}),\langle\phi, \omega\rangle=1\right\} \cup\{0\}\right) .
$$

See Benedetti and Petronio [4, Proposition F.2.2] for a proof.

For $M$ a closed orientable $n$-manifold, Gromov defined the simplicial volume $\|M\|$ to be the Gromov norm of the fundamental class in $H_{n}(M ; \mathbb{R})$. For a manifold with boundary, it is natural to define $\|M, \partial M\|$ to be the Gromov norm of the fundamental class in $H_{n}(M, \partial M ; \mathbb{R})$. It is clear from the definition that this volume only depends on the homotopy type of $(M, \partial M)$. For closed hyperbolic manifolds the simplicial volume is proportional to the volume. More generally, if $M$ is compact, and the interior of $M$ admits a complete finite volume hyperbolic metric of volume Vol, then

$$
\|M, \partial M\|=\frac{1}{v_{n}} \mathrm{Vol},
$$

where $v_{n}$ is the volume of a hyperbolic regular ideal simplex of dimension $n$. (See [4, C.4] for the closed case; we show how to extend to the finite volume case in Appendix A.)

In [31], Yamaguchi extended the notion of simplicial volume to orientable pseudomanifolds, defined as follows.

Definition 2.1 (Yamaguchi [31, Definition 2.1]) A locally compact, Hausdorff, locally homologically $n$-connected space $X$ is called an oriented $n$-pseudomanifold if it contains a closed subset $S$ (the singular set) so that: 
(i) $X \backslash S$ is an orientable $n-$ manifold,

(ii) $\operatorname{dim}(S) \leq n-1$, and

(iii) $\operatorname{dim}(S \cap \overline{X \backslash S}) \leq n-2$.

Remark 2.2 In this definition, we allow the possibility that $S$ is not "really" singular. For example, $X$ could be a manifold and $S$ a codimension 2 submanifold. This is important in applying Proposition 2.3 to $2 \pi$-fillings which happen to be manifolds, as in Corollary 2.5 below.

In [31, Proposition 2.2], Yamaguchi shows that an oriented $n$-pseudomanifold $X$ has a fundamental class $[X]$ in $H_{n}(X)=H_{n}\left(\pi_{1}(X)\right)$ so that the orientation cocycle $\beta \in H^{n}(X)$ is dual to this class. For such a pseudomanifold $X$, the simplicial volume of $X$ is defined to be the Gromov norm of $[X]$, and written $\|X\|$.

If $X$ is compact with CAT $(-1)$ universal cover, then $X$ is aspherical and $\pi_{1}(X)$ is (word) hyperbolic (see Gromov [12]). In this case the orientation cocycle is cohomologous to a bounded cocycle by the main theorem of the paper [21] by Mineyev, and so $\|X\|>0$ by the duality equation (3).

If $X$ is compact and the universal cover is CAT( 0$)$ with isolated flats, then $X$ is again aspherical, but $\pi_{1}(X)$ is only relatively hyperbolic, relative to fundamental groups of images of flats in $X$ (see Hruska and Kleiner [17]). In this case, we will need the following corollary of a theorem of Mineyev-Yaman:

Proposition 2.3 Let $M$ be a closed orientable aspherical $n$-dimensional pseudomanifold with singular set $V \subset M$ which is a disjoint union of aspherical components $V=\cup_{i=1}^{m} V_{i}$. Suppose that $\pi_{1}(M)$ is hyperbolic relative to the collection of subgroups $\left\{\pi_{1}\left(V_{i}\right) \mid i \in\{1, \ldots, n\}\right\}$. Then $\|M\|>0$.

Proof The inclusion of pairs $(M, \varnothing) \rightarrow(M, V)$ gives a natural map

$$
H^{n}(M, V ; \mathbb{R}) \rightarrow H^{n}(M ; \mathbb{R}) .
$$

Since $\operatorname{dim}(V \cap \overline{M \backslash V}) \leq n-2$, the Mayer-Vietoris exact sequence shows this map to be an isomorphism (see Yamaguchi [31, Proposition 2.2]). The main result of Mineyev-Yaman [22] implies that the map

$$
H_{b}^{n}\left(\pi_{1}(M),\left\{\pi_{1}\left(V_{i}\right)\right\}_{i=1}^{m} ; \mathbb{R}\right) \rightarrow H^{n}\left(\pi_{1}(M),\left\{\pi_{1}\left(V_{i}\right)\right\}_{i=1}^{m} ; \mathbb{R}\right)
$$

is surjective, since $\pi_{1}(M)$ is hyperbolic relative to the fundamental groups of the components of $V$. Since $M$ and the components of $V$ are aspherical, this is the same as saying that the map

$$
H_{b}^{n}(M, V ; \mathbb{R}) \rightarrow H^{n}(M, V ; \mathbb{R})=H^{n}(M ; \mathbb{R})
$$


is surjective. It follows that the orientation cocycle of $M$ is cohomologous to a bounded cocycle $\alpha$. (The cocycle moreover can be chosen to vanish on chains in $V$, but we don't use this.) Equation (3) gives $\|M\| \geq \frac{1}{\|\alpha\|_{\infty}}>0$.

Applying Theorem 1.4, we immediately obtain the lower bound of Theorem 1.5.

Corollary 2.4 If $M\left(T_{1}, \ldots, T_{m}\right)$ is a $2 \pi$-filling as in Theorem 1.5, then

$$
\left\|M\left(T_{1}, \ldots, T_{m}\right)\right\|>0 .
$$

The special case of nonpositively curved manifolds obtained by $2 \pi-$ filling of $\geq$ 4-dimensional hyperbolic manifolds has been studied before us by Schroeder [28], Anderson [1] and others. Ivanšić describes in [18] a particular cusped hyperbolic manifold which is the complement of five linked 2-tori in the 4-sphere. Ratcliffe and Tschantz point out in [27] that appropriately chosen $2 \pi$-fillings of this hyperbolic manifold are homology 4-spheres. In [3, Question 7.2], Belegradek asked if such fillings have positive simplicial volume. As a special case of Corollary 2.4 above, we give an affirmative answer.

Corollary 2.5 Every manifold obtained by $2 \pi$-filling of an orientable hyperbolic $n-$ manifold with torus cusps has positive simplicial volume. In particular, the aspherical homology 4-spheres constructed by Ratcliffe and Tschantz have positive simplicial volume.

Theorem 1.5 gives further information (an upper bound) on the simplicial volume.

\subsection{Symmetrization}

It will be convenient in the arguments of Section 3 to assume that all chains considered are symmetric. Here we say what this means and justify the assumption.

Consider the abstract $n$-simplex $\Delta^{n}$. The symmetric group $S_{n+1}$ acts in an obvious way on the vertices, and any permutation $p \in S_{n+1}$ extends to $\Delta^{n}$ as an affine transformation.

Let $X$ be some space. Any singular simplex $\sigma: \Delta^{n} \rightarrow X$ can be altered by precomposition with some $p \in S_{n+1}$ and the singular simplices $\sigma$ and $\sigma \circ p$ are generally different, though if $p$ is an odd permutation and $q$ is an even one $(\operatorname{son} \operatorname{sgn}(p)=-1$ and $\operatorname{sgn}(q)=1$ ), we'd really like to think of $\sigma \circ p$ as being the same as $\sigma \circ q$ or $\sigma$, just with opposite orientation. We would also like to avoid the annoying bookkeeping 
which is standard when dealing with simplices. We therefore define the symmetrization map

$$
S: C_{*}(X ; \mathbb{R}) \rightarrow C_{*}(X ; \mathbb{R})
$$

on singular $n$-simplices $\sigma$ by

$$
S(\sigma)=\frac{1}{(n+1) !} \sum_{p \in S_{n+1}} \operatorname{sgn}(p)(\sigma \circ p),
$$

and extend linearly over $C_{*}(X ; \mathbb{R})$.

Lemma 2.6 $S$ is a chain map, chain homotopic to the identity. Moreover $\|S(c)\|_{1} \leq$ $\|c\|_{1}$ for any chain $c$.

Proof It is evident that norm does not increase. The chain homotopy is described in Appendix B.

Define $C_{*}^{S}(X ; R)$ to be the subcomplex of $C_{*}(X ; R)$ consisting of chains in the image of $S$; the transformation $S$ restricts to the identity on this subcomplex. Lemma $2.6 \mathrm{im}-$ plies that in computing Gromov norms, we may as well restrict attention to $C_{*}^{S}(X ; R)$.

Suppose $X$ has the structure of a simplicial complex (or more generally just a CW complex so that each cell is a simplex, and so that each restriction of a gluing map to a face is affine) with triangulation $\mathcal{T}$.

Definition 2.7 We call a singular simplex $\sigma: \Delta^{n} \rightarrow X$ affine if $\sigma=\tau \circ a$ where $a: \Delta^{n} \rightarrow \Delta^{k}$ is some affine surjection taking vertices to vertices, and $\tau$ is the characteristic map of some $k$-simplex of $\mathcal{T}$. A linear combination of affine simplices is an affine chain, and so on.

Remark 2.8 There is a nice inclusion

$$
\mathcal{C}_{*}^{\mathcal{T}}(X ; \mathbb{R}) \stackrel{i}{\longrightarrow} C_{*}^{S}(X ; \mathbb{R})
$$

of simplicial chains $\mathcal{C}_{*}^{\mathcal{T}}(X ; \mathbb{R})$ given by sending a simplex of $X$ to the symmetrization of its characteristic map. This gives a way to think of simplicial chains as symmetric affine singular chains.

In fact, the image of the map $i$ from Remark 2.8 contains all of the symmetric affine simplices; the symmetrization of an affine simplex which drops dimension must vanish: 
Lemma 2.9 Let $X$ have a triangulation $\mathcal{T}$, and suppose that $\sigma$ is an affine singular $n$-simplex with image in the $(n-1)$-skeleton of $X$. Then $S(\sigma)=0$.

Proof Since $\sigma$ has image in the $(n-1)$-skeleton, there must be a pair of vertices $\left\{v_{i}, v_{j}\right\}$ of the standard simplex $\Delta^{n}$ which are identified by $\sigma$. Let $p$ be a permutation of the vertices of $\Delta^{n}$, and let $\tau$ be the transposition switching $v_{i}$ and $v_{j}$. The maps $\sigma \circ p$ and $\sigma \circ \tau \circ p$ are the same as singular simplices, but the permutations $p$ and $\tau \circ p$ have opposite signs, so the terms $\operatorname{sgn}(p)(\sigma \circ p)$ and $\operatorname{sgn}(\varnothing \circ p)(\sigma \circ \tau p)$ cancel in the sum (5).

\section{Main result}

In this section we prove the main result, Theorem 1.5. The strategy is to modify an efficient relative fundamental cycle for $(M, \partial M)$ to a fundamental cycle on the filling, without increasing norm by too much.

Lemma 3.1 Let $X$ be a topological space. Let $c \in C_{n}(X ; \mathbb{R})$ be homologous to a rational chain, and let $\epsilon>0$. Then there is some $c^{\prime} \in C_{n}(X ; \mathbb{Q})$ homologous to $c$ so that $\left\|c^{\prime}-c\right\|_{1}<\epsilon$. If $c$ is symmetric, $c^{\prime}$ can be chosen to be symmetric.

Proof By assumption, there is some $f \in C_{n+1}(X ; \mathbb{R})$ so that $c+\partial f \in C_{n}(X ; \mathbb{Q})$. Since $C_{n+1}(X ; \mathbb{Q})$ is dense in $C_{n+1}(X ; \mathbb{R})$, there is some $f^{\prime} \in C_{n+1}(X ; \mathbb{Q})$ with $\left\|f-f^{\prime}\right\|<\frac{\epsilon}{n+2}$. The chain $c^{\prime}=c+\partial\left(f-f^{\prime}\right)$ satisfies $\left\|c^{\prime}-c\right\|<\epsilon$, since $\left\|\partial_{n+1}\right\|=$ $n+2$.

If $c$ is symmetric, we can take $f$ and $f^{\prime}$ to be symmetric in the above argument, and it will follow that $c^{\prime}$ is symmetric.

Definition 3.2 Let $\mathcal{T}$ be a finite triangulation of a space $X$, and let $\mathcal{T}^{\prime}$ be its barycentric subdivision. The (closed) star neighborhood of a simplex $\tau$ of $\mathcal{T}$ is the union of those simplices of the subdivision $\mathcal{T}^{\prime}$ intersecting $\tau$. A point in the interior of an $n$-simplex of $X$ lies in at least one closed star neighborhood of a vertex, and in at most $n+1$ such closed stars.

The open star neighborhood of a simplex is the interior of its star neighborhood. (So the open star neighborhood of a simplex does not include its proper faces.) The open star neighborhoods of the simplices of $\mathcal{T}$ form an open cover of $X$.

Let $\sigma: \Delta^{n} \rightarrow X$ be a singular simplex. We say $\sigma$ is small with respect to $\mathcal{T}$ if the image of $\sigma$ is contained entirely in the open star of some vertex of $\mathcal{T}^{\prime}$.

A singular chain will be called small if all its simplices are small. 
Given an order on the vertices of $\mathcal{T}$ there is a canonical way to homotope a small chain to an affine chain, as we describe in the next lemma.

Lemma 3.3 Let $X$ be a space with a (combinatorial) triangulation $\mathcal{T}$, and let $<_{\mathcal{T}}$ be a total order on the vertices of $\mathcal{T}$. Canonically associated to any small singular simplex $\sigma: \Delta^{n} \rightarrow X$ are

(1) a "straightened" affine simplex $a(\sigma)$, and

(2) a homotopy $f_{t}(\sigma)$ so that $f_{0}(\sigma)=\sigma$ and $f_{1}(\sigma)=a(\sigma)$,

so that if $F$ is a face of $\Delta^{n}$, then $a(\sigma \mid F)=a(\sigma) \mid F$ and $f_{t}(\sigma \mid F)=f_{t}(\sigma) \mid F$. (In other words, homotopies on a face depend only on that face, so a cycle remains a cycle throughout the homotopy.) Moreover, if $\sigma$ is invariant under precomposition with an affine map $\tau$, so are $a(\sigma)$ and $f_{t}(\sigma)$.

Proof The idea is illustrated quite well by the figure given in Thurston's notes [29, Proposition 6.5.1]. We give a little more detail. (The figure given there is not of a combinatorial triangulation. In that case a total order on the "corners" of the simplices must be given, rather than on the vertices. We avoid this complication here by assuming a combinatorial triangulation.)

We begin by defining the straightening $a(\sigma)$. For $x \in X$ define $n(x)$ to be the least vertex $v$ of $\mathcal{T}$ so that $x$ is in the closed star neighborhood of $v$. Let $\left\{v_{0}, \ldots, v_{n}\right\}$ be the vertices of the standard simplex $\Delta^{n}$. Since $\sigma$ is small, the image of $\sigma$ lies in the open star neighborhood of some simplex $\tau$ of $\mathcal{T}$. (In fact there may be several choices for $\tau$, but the maps $a(\sigma)$ and $f_{t}(\sigma)$ we define will not depend on the choice.) It follows that $n\left(\sigma\left(v_{i}\right)\right)$ is a vertex of $\tau$ for each $i$. We define $a(\sigma)\left(v_{i}\right)=n\left(\sigma\left(v_{i}\right)\right)$ and then extend to an affine map $a(\sigma): \Delta^{n} \rightarrow \tau$. It is clear that if $F$ is a face of $\Delta^{n}$ we have $a(\sigma \mid F)=a(\sigma) \mid F$.

We now must define the homotopies $f_{t}(\sigma)$. For each $x \in \Delta^{n}$, we let $\tau(x)$ be the unique simplex of $\mathcal{T}$ so that

(1) $\sigma(x) \in \tau(x)$, and

(2) $\tau(x)$ has minimal dimension subject to (1).

Since $\tau(x)$ lies in the open star neighborhood of $\tau$, the simplex $\tau$ is a face of $\tau(x)$. Thus both $\sigma(x)$ and $a(\sigma)(x)$ lie in $\tau(x)$. Convex combination is well-defined for points in the same simplex, so we may take, for $t \in[0,1]$,

$$
f_{t}(\sigma)(x)=(1-t) \sigma(x)+t a(\sigma)(x) .
$$


The reader may check that $f_{t}$ is well-defined, continuous, and satisfies the property $f_{t}(\sigma \mid F)=f_{t}(\sigma) \mid F$ for each face $F$ of $\Delta^{n}$.

Finally, we note that if $\tau$ is some affine map from $\Delta^{n}$ to itself so that $\sigma \circ \tau=\sigma$, then $a(\sigma \circ \tau)=a(\sigma)$ and therefore $f_{t}(\sigma \circ \tau)=f_{t}(\sigma)$ for all $t$.

Lemma 3.4 Let $N$ be a manifold, and let $\mathcal{T}$ be a triangulation of $\partial N$. If $c \in C_{k}(N ; \mathbb{R})$ is a relative singular cycle in $(N, \partial N)$ so that $\partial c$ is small with respect to $\mathcal{T}$, then $c$ is (relatively) homologous to $c^{\prime}$ with $\left\|c^{\prime}\right\|_{1} \leq\|c\|_{1}$ and $\partial c^{\prime}$ an affine chain. If $c$ was symmetric, then so is $c^{\prime}$.

Proof The chain $c$ is a finite linear combination $c=\sum_{i=1}^{m^{\prime}} \lambda_{i} \sigma_{i}$ of singular simplices $\sigma_{i}: \Delta^{k} \rightarrow N$. The idea is to "preassemble" the simplices to get a map $\phi: K_{c} \rightarrow N$ from a certain CW complex $K_{c}$, so that $c$ is $\phi_{\#}$ of a linear combination of characteristic maps. We can use Lemma 3.3 to homotope the "boundary" of $K_{c}$ to a simplicial map, and then apply the homotopy extension property of $\mathrm{CW}$ pairs.

More precisely, let $J=\left\{1, \ldots m^{\prime}\right\} / \sim$, where $i \sim j$ if $\sigma_{i}=\sigma_{j} \circ q$ for some permutation $q$. We define

$$
K_{c}=\left(\coprod_{[i] \in J} \Delta_{[i]}^{k}\right) / \sim
$$

where $x_{1} \sim x_{2}$ whenever there are $(k-1)$-dimensional faces $F_{1} \subset \Delta_{\left[i_{1}\right]}^{k}$ and $F_{2} \subset \Delta_{\left[i_{2}\right]}^{k}$ satisfying

(1) $x_{j} \in F_{i}$ for $j \in\{1,2\}$, and

(2) there is an affine map $\tau: F_{1} \rightarrow F_{2}$ so that $\tau\left(x_{1}\right)=x_{2}$ and $\sigma_{i_{1}} \mid F_{1}=\sigma_{i_{2}} \circ \tau$.

That is, if two singular simplices agree on a codimension 1 face, we glue the corresponding faces together in $K_{c}$. Moreover, if a codimension 1 face is unchanged by precomposing with some isometry, we identify the face to itself by the corresponding symmetry. We thus obtain a CW complex $K_{c}$ and a map $\phi: K_{c} \rightarrow N$ given by $\phi(x)=\sigma_{i}(x)$ where $x \in \Delta_{[i]}^{k}$. In the complex $K_{c}$ we can canonically build a chain $\widetilde{c}$ made up of "characteristic maps" $\Delta_{[i]}^{k} \rightarrow K_{c}$ so that

$$
c=\phi_{\sharp}(\widetilde{c}) \text {. }
$$

If $c$ was symmetric, then so is $\tilde{c}$.

The boundary $\partial \widetilde{c}$ is supported in some minimal subcomplex of $K_{c}$, which we'll refer to as $\partial K_{c}$. Because $c$ is a relative cycle, the map $\phi$ described in the last paragraph is really a map of pairs

$$
\phi:\left(K_{c}, \partial K_{c}\right) \rightarrow(N, \partial N) .
$$


After fixing some total order on the simplices of $\mathcal{T}$, Lemma 3.3 gives a homotopy in $\partial N$ of $\phi \mid \partial K_{c}$ to a "simplicial" map $\psi$; this map is simplicial in the sense that if $F$ is any face of one of the simplices $\Delta_{[i]}^{k}$ used to define $K_{c}$, and if $\iota_{F}$ is the inclusion map, then $\phi \circ \iota_{F}$ is affine, with image in a single simplex of $\mathcal{T}$.

By the homotopy extension property of CW pairs, the homotopy from $\phi \mid \partial K_{c}$ to $\psi$ can be extended to all of $K_{c}$. Let

$$
\Phi_{t}: K_{c} \rightarrow N, t \in[0,1]
$$

be this homotopy, with $\Phi_{0}=\phi$. The chain

$$
c^{\prime}=\left(\Phi_{1}\right)_{\sharp}(\widetilde{c})
$$

has all the properties we want.

We need to show that tori can be triangulated so that every simplex of diameter much smaller than the injectivity radius is small. Say that a triangulation of a torus is $K-$ fat if every singular simplex of diameter less than $K$ is small with respect to that triangulation.

Lemma 3.5 For each $d \geq 1$ there is a constant $0<K_{d}<1$ so that every flat torus of dimension $d$ with injectivity radius $R$ has a $\left(K_{d} R\right)-$ fat triangulation with $t_{d}=2^{d} d$ ! simplices.

Proof Let $T$ be a flat $d$-torus, which we can think of as $\mathbb{R}^{d} / \Lambda$, where $\Lambda$ is some rank $d$ lattice. Every such lattice has a Lovász-reduced basis (see Lenstra, Lenstra and Lovász [19]) $\mathcal{B}=\left\{\mathbf{v}_{1}, \ldots, \mathbf{v}_{d}\right\}$. For $k \in\{1, \ldots, d\}$ define $\theta_{k}$ to be the angle made by $\mathbf{v}_{k}$ with the subspace of $\mathbb{R}^{d}$ spanned by the rest of the basis. Babai [2] showed that, for all $k$,

$$
\sin \left(\theta_{k}\right) \geq\left(\frac{\sqrt{2}}{3}\right)^{d} .
$$

Any basis $\mathcal{B}$ of $\Lambda$ gives rise in a canonical way to a triangulation $\mathcal{T}_{\mathcal{B}}$ of $T$ with $t_{d}$ simplices given by the first barycentric subdivision of the parallelepiped spanned by $\mathcal{B}$.

Let $V$ be the set of all ordered bases of lattices in $\mathbb{R}^{d}$, topologized as a subset of $\mathbb{R}^{d^{2}}$, and let $F: V \rightarrow \mathbb{R}_{+}$be the function which sends $\mathcal{B}$ to the least real $k$ so that $\mathcal{T}_{\mathcal{B}}$ is $k$-fat. The number $F(\mathcal{B})$ is also the least number $k$ so that triangulation of $\mathbb{R}^{d}$ covering $T_{\mathcal{B}}$ is $k$-fat. We note the following useful properties of $F$ :

(1) $F$ is continuous and positive everywhere on $V$.

(2) If $\lambda \in \mathbb{R}_{+}$and $\mathcal{B} \in V$, then $F(\lambda \mathcal{B})=\lambda F(\mathcal{B})$. 
(3) If there is a length-decreasing map from $\mathcal{B}$ to $\mathcal{B}^{\prime}$ then $F(\mathcal{B}) \geq F\left(\mathcal{B}^{\prime}\right)$.

Let $U \subset V$ be the set of bases consisting of unit vectors, any two of which make an angle $\theta$ with $\sin (\theta) \geq\left(\frac{\sqrt{2}}{3}\right)^{d}$. Since $U$ is compact, $F$ takes some minimum, positive value $L$ on $U$. We will show that every torus of injectivity radius $\geq 1 / 2$ has a triangulation which is $L$-fat. Linearity of $F$ (property (2) above) then implies the lemma for $K_{d}=2 L$.

Let $T=\mathbb{R}^{d} / \Lambda$ be a torus of injectivity radius at least $1 / 2$. A Lovász-reduced basis $\mathcal{B}=\left\{\mathbf{v}_{1}, \ldots, \mathbf{v}_{d}\right\}$ for $\Lambda$ has all angles between basis vectors within the interval

$$
\left[\sin ^{-1}\left(\left(\frac{\sqrt{2}}{3}\right)^{d}\right), \pi-\sin ^{-1}\left(\left(\frac{\sqrt{2}}{3}\right)^{d}\right)\right],
$$

by Babai's result. Since the injectivity radius of $T$ is at least $1 / 2$, there is a distancedecreasing map from $\mathcal{B}$ to a basis in $U$, namely

$$
\mathcal{B}^{\prime}=\left\{\frac{\mathbf{v}_{1}}{\left\|\mathbf{v}_{1}\right\|}, \ldots, \frac{\mathbf{v}_{d}}{\left\|\mathbf{v}_{d}\right\|}\right\} .
$$

Property (3) above tells us that $F(\mathcal{B}) \geq F\left(\mathcal{B}^{\prime}\right) \geq L$, so the triangulation $\mathcal{T}_{\mathcal{B}}$ is $L$-fat, and the proof of the lemma is completed.

Lemma 3.6 For each $d \geq 1$ and each $s$ there is a constant $K(d, s)$ so that any null-homologous real singular $s$-cycle $z$ in a $d$-dimensional torus bounds a singular $(s+1)$-chain $c$ with

$$
\|c\|_{1} \leq K(d, s)\|z\|_{1}
$$

Proof We follow the proof of Thurston [29, Proposition 6.5.1], which discusses the case $d=s=2$. Let $T$ be a $d$-dimensional torus, and let $z$ be an $s$-cycle. Since there are only finitely many singular simplices in $z$, their lifts to the universal cover $\mathbb{R}^{d}$ have diameters bounded by some $D>0$. Let $K_{d}$ be the constant from Lemma 3.5, and let $\widetilde{T} \stackrel{\pi}{\longrightarrow} T$ be some cover of $T$ with injectivity radius bigger than $D / K_{d}$. By Lemma 3.5 , there is a triangulation $\mathcal{T}$ of $\widetilde{T}$ with $t_{d}$ simplices so that if $\widetilde{\sigma}: \Delta^{s} \rightarrow \widetilde{T}$ is any lift of a singular simplex appearing in $z$, then $\widetilde{\sigma}$ is small with respect to $\mathcal{T}$ (in the sense of Definition 3.2). The proof of Lemma 3.5 shows that the combinatorics of this triangulation are independent of the particular chain $z$ and the cover $\widetilde{T}$.

Write $C_{*}(X)$ for the real singular chain complex of $X$. There is a transfer map

$$
\text { trans: } C_{*}(T ; \mathbb{R}) \rightarrow C_{*}(\tilde{T} ; \mathbb{R})
$$

which sends any singular simplex to the average of its lifts. The map trans is an isometric embedding with respect to the $l^{1}$ norm. The map $\pi_{\sharp}: C_{*}(\widetilde{T} ; \mathbb{R}) \rightarrow C_{*}(T ; \mathbb{R})$ induced by the covering is norm decreasing and ( $\pi_{\sharp} \circ$ trans) is the identity map. 
By the choice of $\tilde{T}$ and $\mathcal{T}$, the chain $\operatorname{trans}(z)$ is small. By Lemma 3.3, the simplices of $\operatorname{trans}(z)$ can be homotoped simultaneously and consistently to the affine singular simplices making up the singular chain $a(\operatorname{trans}(z))$. Triangulating this homotopy in a standard way, we find that $\operatorname{trans}(z)-a(\operatorname{trans}(z))$ bounds an $(s+1)-$ chain $c_{1}$ in $T$, so that

$$
\left\|c_{1}\right\|_{1} \leq C_{1}\|z\|_{1}
$$

for some constant $C_{1}$ depending on the dimension $s$ but otherwise independent of $z$. The space of affine real singular $s$-boundaries $B$ is finite dimensional, and only depends on the combinatorics of the triangulation $\mathcal{T}$.

The affine filling norm $\|b\|_{\text {fill }}$ on $B$ is defined, for a boundary $b \in B$, to be the smallest $l^{1}$-norm of an affine $(s+1)$-chain bounded by $b$. Let

$$
C_{2}(d, s)=\sup \left\{\|b\|_{\text {fill }} \mid b \in B \text { and }\|b\|_{1}=1\right\},
$$

and note that $C_{2}(d, s)$ only depends on the combinatorics of $\mathcal{T}$.

The boundary $a(\operatorname{trans}(z))$ bounds an $(s+1)$-chain $c_{2}$ in $T$ of $l^{1}-$ norm at most $C_{2}(d, s)\|a(\operatorname{trans}(z))\|_{1} \leq C_{2}\|z\|_{1}$. Thus we have

$$
\operatorname{trans}(z)=\partial\left(c_{1}+c_{2}\right)
$$

and

$$
z=\pi_{\sharp}\left(\partial\left(c_{1}+c_{2}\right)\right)=\partial\left(\pi_{\sharp}\left(c_{1}+c_{2}\right)\right)
$$

and

$$
\left\|\pi_{\sharp}\left(c_{1}+c_{2}\right)\right\|_{1} \leq\left\|c_{1}+c_{2}\right\|_{1} \leq\left(C_{1}(s)+C_{2}(d, s)\right)\|z\|_{1} .
$$

We now prove the main theorem.

Proof of Theorem 1.5 We suppose that $M$ is a compact $n$-manifold with boundary a union of tori, so that the interior admits a complete hyperbolic metric of finite volume. We fix a $2 \pi$-filling $M\left(T_{1}, \ldots, T_{m}\right)$. To prove the theorem we must show

$$
0<\left\|M\left(T_{1}, \ldots, T_{m}\right)\right\| \leq \frac{\operatorname{Vol}(V)}{v_{n}},
$$

where $v_{n}$ is the volume of a regular ideal hyperbolic $n$-simplex.

The lower bound follows from Mineyev-Yaman [22] and is given above in Corollary 2.4.

We now establish the upper bound. The strategy is to first choose a symmetric representative $c_{0}$ for the (relative) fundamental class of $(M, \partial M)$ which is close to optimal, and then modify it to obtain a fundamental class $c_{0}^{\prime}$ for $M\left(T_{1}, \ldots, T_{m}\right)$. If such a 
modification can be done in such a way that $\left|c_{0}^{\prime}\right|_{1}$ is within some arbitrarily small constant of $\|M, \partial M\|$, then we will have shown

$$
\left\|M\left(T_{1}, \ldots, T_{m}\right)\right\| \leq\|M, \partial M\| .
$$

The first step is to pass to a rational cycle. Let $\epsilon>0$. Let $c_{0} \in C_{n}(M ; \mathbb{R})$ be a real symmetric singular relative cycle representing $[M, \partial M]$, and satisfying

$$
\left\|c_{0}\right\|_{1}<\|M, \partial M\|+\epsilon
$$

By Lemma 3.1, there is a symmetric rational singular relative cycle $c_{1} \in C_{n}(M ; \mathbb{Q})$ homologous to $c_{0}$ with $\left\|c_{1}-c_{0}\right\|_{1}<\epsilon$.

We now pick a finite cover and triangulation of the boundary so that $\partial c_{1}$ lifts to a chain which is small with respect to that triangulation. Let $t_{n-1}=2^{n-1}(n-1)$ !.

Claim 3.7 We can choose a finite cover $\widetilde{M} \stackrel{\pi}{\longrightarrow} M$ and a triangulation $\mathcal{T}$ of $\partial \widetilde{M}$ satisfying the conditions:

(1) The restriction of $\pi$ to any component of $\partial \widetilde{M}$ has degree bigger than $m / \epsilon$, where $m$ is the number of cusps of $M$.

(2) If trans: $C_{*}(M ; \mathbb{R}) \rightarrow C_{*}(\widetilde{M} ; \mathbb{R})$ is the transfer map, then trans $\left(\partial c_{1}\right)$ is small with respect to $\mathcal{T}$.

(3) Each boundary component of $\widetilde{M}$ is triangulated by $t_{n-1}(n-1)-$ simplices.

Proof The compact manifold $M$ is homeomorphic to $\bar{M}$ as described in the introduction, a hyperbolic manifold minus a union of disjoint horospherical neighborhoods of its cusps. We identify $M$ with $\bar{M}$. Any simplex in the support of $\partial c_{1}$ therefore lifts to $\mathbb{H}^{n}$. Only finitely many such simplices occur, so there some number $r$ bounding the diameter of any such lift. Residual finiteness of $\pi_{1} M$ implies that we can pass to a finite cover $\widetilde{M} \rightarrow M$ so that

(1) Every boundary component of $\widetilde{M}$ has injectivity radius bigger than $r / K_{n-1}$, where $K_{n-1}$ is the constant from Lemma 3.5, and

(2) Every boundary component $N$ of $\widetilde{M}$ has area at least $m / \epsilon$ times more than the area of the component of $\partial M$ covered by $N$.

Lemma 3.5 implies that $\partial \widetilde{M}$ admits an $r$-fat triangulation with $t_{n-1}(n-1)$-simplices per component so that singular simplices lifted from $\partial c$ are all small respect to this triangulation. 
Let $D$ be the degree of the cover coming from Claim 3.7. Since $c_{1}$ is rational and symmetric, $\operatorname{trans}\left(c_{1}\right)$ must also be rational and symmetric. In particular,

$$
\operatorname{trans}\left(c_{1}\right)=\frac{1}{q} c_{2}
$$

for some integral symmetric chain $c_{2} \in C_{*}^{S}(\widetilde{M} ; \mathbb{Z})$ and some positive integer $q$. Homologically $\left[c_{2}\right]=\frac{q}{D}[\widetilde{M}, \partial \widetilde{M}]$ in $H_{n}(\widetilde{M}, \partial \widetilde{M} ; \mathbb{R})$. Since trans: $C_{*}(M ; \mathbb{R}) \rightarrow$ $C_{*}(\widetilde{M} ; \mathbb{R})$ is isometric, $\left\|c_{2}\right\|_{1}=q\left\|c_{1}\right\|_{1}$.

Since trans $\left(\partial c_{1}\right)$ is small with respect to $\mathcal{T}$, the chain $\partial c_{2}$ is also small with respect to $\mathcal{T}$. Using Lemma 3.4, we find a symmetric integral chain $c_{3}$ with $\left\|c_{3}\right\|_{1} \leq\left\|c_{2}\right\|_{1}$ so that $c_{3}$ is (relatively) homologous to $c_{2}$ and so that $\partial c_{3}$ is an affine chain, that is, $\partial c_{3} \in C_{n-1}^{\mathcal{T}}(\partial \widetilde{M} ; \mathbb{Z})$.

Let $\tilde{N}_{1}, \ldots, \tilde{N}_{p}$ be the boundary components of $\widetilde{M}$. We have $\left[c_{3}\right]=\frac{q}{D}[\widetilde{M}, \partial \widetilde{M}]$ in $H_{n}(\widetilde{M}, \partial \widetilde{M})$ and $\left[\partial c_{3}\right]=\frac{q}{D} \sum_{i=1}^{p}\left[\tilde{N}_{i}\right]$ in $H_{n-1}(\partial \tilde{M})$.

The cycle $\partial c_{3}$ is symmetric, affine and represents $\frac{q}{D}$ times the fundamental class of $\partial \tilde{M}$. By Lemma 2.9, $\partial c_{3}$ has no "degenerate" affine simplices in its support. It follows that $\partial c_{3}$ consists of exactly $\frac{q}{D}$ appropriately oriented copies of every $(n-1)-$ simplex in $\mathcal{T}$.

Each of the $p$ boundary components of $\widetilde{M}$ is triangulated with exactly $t_{n-1}$ simplices of dimension $n-1$. We therefore have

$$
\left\|\partial c_{3}\right\|_{1}=p t_{n-1} \frac{q}{D} .
$$

For each $i$ between 1 and $n$, the $i$ th boundary component of $M$ is covered by $p_{i}$ boundary components of $\widetilde{M}$, so that $p=\sum_{i} p_{i}$. The degrees of these components are $d_{i 1}, \ldots, d_{i p_{i}}$, and $\sum_{j} p_{i j}=D$ for each $i$. Since each $d_{i j} \geq \frac{m}{\epsilon}$, we get

$$
D \geq p_{i} \frac{m}{\epsilon} \text { for all } i,
$$

which implies that $m D \geq p \frac{m}{\epsilon}$, and so $p \leq \epsilon D$. We therefore obtain

$$
\left\|\partial c_{3}\right\|_{1} \leq \epsilon t_{n-1} q .
$$

Projecting $c_{3}$ back down to $M$ gives a chain $c_{4}$ with $\left[c_{4}\right]=q[M, \partial M]$ in relative homology, and with

$$
\left\|c_{4}\right\|_{1} \leq\left\|c_{3}\right\|_{1} \leq\left\|c_{2}\right\|_{1}=q\left\|c_{1}\right\|_{1},
$$

and with

$$
\left\|\partial c_{4}\right\|_{1} \leq\left\|\partial c_{3}\right\|_{1} \leq \epsilon t_{n-1} q .
$$


Dividing by $q$ we obtain a fundamental cycle $c=\frac{1}{q} c_{4}$ for $[M, \partial M]$ satisfying $\|c\|_{1} \leq$ $\|M, \partial M\|+2 \epsilon$ and $\|\partial c\|_{1} \leq t_{n-1} \epsilon$.

Now we will take this relative cycle $c$ for $[M, \partial M]$, and modify it to an honest cycle in $M\left(T_{1}, \ldots, T_{m}\right)$. We start by pushing it forward from $M$ to $M\left(T_{1}, \ldots, T_{m}\right)$ by a map of pairs

$$
h:(M, \partial M) \rightarrow\left(M\left(T_{1}, \ldots, T_{m}\right), Z\right),
$$

where $Z$ is the singular set of $M\left(T_{1}, \ldots, T_{m}\right)$ and $h$ is the quotient map described in Definition 1.3 . (Here we are implicitly identifying $M$ with the space $\bar{M}$ described before Definition 1.3.) Let $\partial h$ be $h$ restricted to $\partial M$. The chain $(\partial h)_{\sharp}(\partial c)$ is an $(n-1)-$ cycle in $Z$. Lemma 3.6 implies there is some $K$ depending only on $n$ so that $(\partial h)_{\sharp}(\partial c)$ can be filled in $Z$ with a $n$-chain $c^{\prime}$ satisfying $\left\|c^{\prime}\right\|_{1} \leq K\left\|(\partial h)_{\sharp}(\partial c)\right\|_{1} \leq K t_{n-1} \epsilon$. It is easy to check that $h_{\sharp} c-c^{\prime}$ is a fundamental cycle for $M\left(T_{1}, \ldots, T_{m}\right)$. Moreover, we have

$$
\left\|h_{\sharp} c-c^{\prime}\right\|_{1} \leq\|M, \partial M\|+\left(2+K t_{n-1}\right) \epsilon .
$$

Letting $\epsilon$ tend to zero, we have established

$$
\left\|M\left(T_{1}, \ldots, T_{m}\right)\right\| \leq\|M, \partial M\| .
$$

An application of the proportionality theorem for finite volume hyperbolic manifolds (Theorem A.1) gives the upper bound

$$
\left\|M\left(T_{1}, \ldots, T_{m}\right)\right\| \leq \frac{\operatorname{Vol}(V)}{v_{n}} .
$$

\section{Appendix A A proportionality theorem}

The purpose of this appendix is to establish the following proportionality theorem.

Theorem A.1 Let $M$ be a manifold with boundary, so that the interior $V$ of $M$ admits a complete hyperbolic metric of volume $\operatorname{Vol}(V)<\infty$. Then

$$
\|M, \partial M\|=\frac{\operatorname{Vol}(V)}{v_{n}} .
$$

Thurston gives a proof in dimension 3 of a version of Theorem A.1 in his lecture notes [29, 6.5.4], where the norm $\|M, \partial M\|$ is replaced by one coming from measure homology. There is a natural map from singular homology to measure homology, which at least does not increase norm. Zastrow [32] and Hansen [15] independently showed this map to be an isomorphism of vector spaces for any $\mathrm{CW}$ pair, leaving open the question of whether it was an isometry. Löh [20] proved that the map from 
(absolute) singular homology to measure homology is an isometric isomorphism for all connected CW complexes. Since it is not entirely clear whether measure homology is isometric to singular homology in the relative case, we give a proof of Theorem A.1 which avoids measure homology. Such a proof can also be obtained as a special (and simpler) case of the arguments in Frigerio-Pagliantini [7]. Some related ideas may be found in Francaviglia [6].

Our proof of Theorem A.1 follows closely Benedetti and Petronio's proof of [4, C.4], which covers the case of $\partial M=\varnothing$. As some parts of our proof are identical to steps in [4], we refer to that text for some details. The proof in [4] uses ordinary real singular chains, rather than symmetrized ones, so we do not symmetrize our chains here.

We use the following notation for parts of the manifold $V$ : For $I \subseteq \mathbb{R}_{>0}$, we let $V_{I}$ be the subset of $V$ consisting of points $x \in V$ where the injectivity $\operatorname{radius} \operatorname{inj}(V, x)$ is in $I$.

Definition A.2 Let $M$ be a Riemannian $n$-manifold, and let $\sigma: \Delta^{n} \rightarrow M$ be a smooth singular simplex. The algebraic volume of $\sigma$, written $\operatorname{algvol}(\sigma)$ is the integral over $\Delta^{n}$ of the pullback of the volume form on $M$. The algebraic volume is extended to smooth chains by linearity.

Obtaining the correct lower bound for simplicial volume is fairly easy.

Lemma A.3 $\|M, \partial M\| \geq \frac{\operatorname{Vol}(V)}{v_{n}}$.

Proof This direction is Thurston's "straightening" argument [29, 6.5.4]. For any sufficiently small $\epsilon>0$, the subset $V_{(0, \epsilon]}$ is an open neighborhood of the cusps of $V$. Collapsing the components of $V_{(0, \epsilon]}$ to their boundaries gives a homotopy equivalence of pairs $\left(V, V_{(0, \epsilon]}\right) \rightarrow(M, \partial M)$. Since the Gromov norm of a (relative) homology class is homotopy invariant, we can use chains $\left(V, V_{(0, \epsilon]}\right)$ to compute $\|M, \partial M\|$.

The straightening map (see Benedetti and Petronio [4] or Ratcliffe [26] for a precise definition) is a chain map

$$
\text { str: } C_{*}(V) \rightarrow C_{*}(V),
$$

chain homotopic to the identity, and taking each singular simplex to a totally geodesic simplex with the same vertices. The map str preserves the subspace $C_{*}\left(V_{(0, \epsilon]}\right)$ (because horoballs are convex), as does the chain homotopy between str and the identity, so str induces a (norm-decreasing) chain homotopy equivalence of $C_{*}\left(V, V_{(0, \epsilon]}\right)$ to itself. It follows that we need only consider straight (and therefore smooth) chains. 
If $z=\sum_{i}^{k} \lambda_{i} \sigma_{i}$ is a real smooth chain representing the relative fundamental class $\left[V, V_{(0, \epsilon]}\right]$, then

$$
\operatorname{algvol}(z)=\sum_{i}^{k} \lambda_{i} \operatorname{algvol}\left(\sigma_{i}\right)
$$

The absolute value $|\operatorname{algvol}(z)|$ is at least as big as $\operatorname{Vol}\left(V_{[\epsilon, \infty)}\right)$, the volume of the thick part of $V$.

Let $z_{\epsilon}=\sum_{i}^{k} \lambda_{i} \sigma_{i}$ be a straight real singular chain representing $\left[V, V_{(0, \epsilon]}\right]$ which $\epsilon$-nearly realizes $\|M, \partial M\|$, that is, so that

$$
\left\|z_{\epsilon}\right\|_{1} \leq\|M, \partial M\|+\epsilon \text {. }
$$

We then have

$$
\operatorname{Vol}\left(V_{[\epsilon, \infty)}\right) \leq\left|\operatorname{algvol}\left(z_{\epsilon}\right)\right| \leq \sum_{i=1}^{k}\left|\lambda_{i}\right| v_{n}=\left\|z_{\epsilon}\right\|_{1} v_{n} \leq(\|M, \partial M\|+\epsilon) v_{n}
$$

Letting $\epsilon$ tend to zero in (8) yields the lemma.

It is worth noting that essentially the same proof establishes the bound of Lemma A.3 for measure cycles.

To obtain the upper bound for simplicial volume, we must construct chains representing the fundamental class which are "close" to the smeared chains from measure homology. The argument from [4] in the closed case uses rather strongly that there is a compact (and therefore finite diameter) fundamental domain. We work around this by chopping the fundamental domain into pieces of bounded diameter.

Proposition A.4 $\|M, \partial M\| \leq \frac{\operatorname{Vol}(V)}{v_{n}}$

Proof We suppose that $V$ is an orientable finite volume hyperbolic $n$-manifold with $m$ cusps, homeomorphic to the interior of $M$. Let $\Gamma<\operatorname{Isom}\left(\mathbb{H}^{n}\right)$ be the fundamental group of $V$, so that $\mathbb{H}^{n} / \Gamma=V$. Let $D$ be the closure of a convex fundamental domain for the action of $\Gamma$ on $\mathbb{H}^{n}$, and let $\pi: D \rightarrow V$ be the quotient.

There is a number $\mu>0$ so that $V_{(0, \epsilon]}$ has exactly $m$ connected components for any $\epsilon \leq \mu$. Let $P_{0} \subseteq V$ be equal to $V_{(\mu, \infty)}$, and choose some $x_{0} \in P_{0}$. The complement of $P_{0}$ in $V$ is a union of cusp neighborhoods $C_{1}, \ldots, C_{n}$.

Let $D_{0}=\pi^{-1}\left(P_{0}\right) \subset D$, and choose $\tilde{x}_{0}$ in the interior of $D_{0}$. Let $x_{0}=\pi\left(\tilde{x}_{0}\right) \in P_{0}$. For each $l \in \mathbb{N}$, and each $j \in\{1, \ldots, m\}$, we let

$$
P_{j, l}=\left\{x \in V \mid l-1 \leq d\left(x, P_{0}\right) \leq l\right\} \cap C_{j},
$$


and let $D_{j, l}=\pi^{-1}\left(P_{j, l}\right) \subset D$. We will refer to any $\Gamma$-translate of $D_{0}$ or $D_{j, l}$ as a piece.

There is some $d>0$ so that $\operatorname{diam}\left(D_{\alpha}\right)<d$ for every piece $D_{\alpha} \subset \mathbb{H}^{n}$. For each $l \in \mathbb{N}$ and each $j \in\{1, \ldots, m\}$, choose $\tilde{x}_{j, l}$ in the interior of $D_{j, l}$ and let $x_{j, l}=\pi\left(\tilde{x}_{j, l}\right)$. The union of the $\Gamma$-orbits of $\tilde{x}_{0}$ and the $\tilde{x}_{j, l}$ can be identified in an obvious way with

$$
\hat{\Gamma}:=\Gamma \cup(\Gamma \times\{1, \ldots, m\} \times \mathbb{N}) .
$$

(This is essentially the 0-skeleton of the "cusped space" of Groves and Manning [14] associated to the pair $\left(\Gamma,\left\{\pi_{1}\left(C_{j}\right)\right\}\right)$.) Vertices of $\widehat{\Gamma}$ have a depth associated to them: The depth of an element of $\Gamma$ is 0 , and the depth of an element of $\Gamma \times\{1, \ldots, m\} \times \mathbb{N}$ is given by the third coordinate. The group $\Gamma$ acts on $\hat{\Gamma}$ by left multiplication in an obvious way, and this action preserves depth.

We let $\Omega$ be the quotient $\Gamma \backslash \hat{\Gamma}^{n+1}$. For $k>0$ an integer, let $\hat{\Gamma}_{k} \subset \hat{\Gamma}$ be the subset of vertices of depth at most $k$. Let $\widetilde{\Omega}_{k}=\widehat{\Gamma}_{k}^{n+1}$, and let $\Omega_{k} \subset \Omega$ be the set of $\Gamma$-orbits in $\tilde{\Omega}_{k}$.

For any element $\omega=\left[\left(y_{0}, \ldots, y_{n}\right)\right] \in \Omega$, there is a unique straight simplex

$$
\sigma_{\omega}: \Delta^{n} \rightarrow V
$$

which is equal to the composition of a simplex $\widetilde{\sigma}_{\omega}: \Delta^{n} \rightarrow \mathbb{H}^{n}$ satisfying $\widetilde{\sigma}_{\omega}\left(v_{i}\right)=y_{i}$ with the projection $\mathbb{H}^{n} \rightarrow V$. (Here $v_{i}$ is the $i$ th vertex of the $n$-simplex $\Delta^{n}$.) As in [4], we define

$\mathcal{S}(R)=\left\{\left(u_{0}, \ldots u_{n}\right) \in\left(\mathbb{H}^{n}\right)^{n+1} \mid d\left(u_{i}, u_{j}\right)=R\right.$, for all $\left.i \neq j\right\}=\mathcal{S}_{+}(R) \sqcup \mathcal{S}_{-}(R)$,

where $\mathcal{S}_{+}$and $\mathcal{S}_{-}$are those tuples which determine positively and negatively oriented straight $n$-simplices in $\mathbb{H}^{n}$, respectively. There is a measure on $\mathcal{S}(R)$ coming from Haar measure on $\operatorname{Isom}\left(\mathbb{H}^{n}\right)$, and we denote this measure by $m$. For

$$
\omega=\left[\left(y_{0}, \ldots, y_{n}\right)\right] \in \Omega,
$$

and $R>0$, we define

$$
\begin{aligned}
& a_{R}^{+}(\omega)=m\left(\left\{\left(u_{0}, \ldots, u_{n}\right) \in \mathcal{S}_{+}(R) \mid u_{i} \text { and } y_{i} \text { lie in the same piece }\right\}\right), \text { and } \\
& a_{R}^{-}(\omega)=m\left(\left\{\left(u_{0}, \ldots, u_{n}\right) \in \mathcal{S}_{-}(R) \mid u_{i} \text { and } y_{i} \text { lie in the same piece }\right\}\right) .
\end{aligned}
$$

Obviously these definitions do not depend on the choice of representative of $\omega$. Moreover, for a given $R$ and $k$, there are only finitely many $\omega \in \Omega_{k}$ for which $a_{R}^{+}(\omega)$ or $a_{R}^{-}(\omega)$ is nonzero. (This is because there are only finitely many elements of $\hat{\Gamma}$ which 
are distance approximately $R$ from a given element of $\widehat{\Gamma}$.) It follows that if we define $a_{R}(\omega):=a_{R}^{+}(\omega)-a_{R}^{-}(\omega)$, then

$$
z_{R, k}=\sum_{\omega \in \Omega_{k}} a_{R}(\omega) \sigma_{\omega}
$$

is a finite real singular $n$-chain in $V$. Moreover, we claim that

$$
z_{R, \infty}=\sum_{\omega \in \Omega} a_{R}(\omega) \sigma_{\omega}
$$

is a locally finite real singular $n$-chain. Indeed, let $p \in V$, and $r>0$. We must show only finitely many simplices in the support of $z_{R, \infty}$ (that is, occurring in the sum (9) with nonzero coefficient) intersect the ball of radius $r$ about $p$. Let $\tilde{p}$ be any point in $\pi^{-1}(p) \subset D$, and note that any simplex $\sigma_{\omega}$ in the support of $z_{R, \infty}$ must lift to one which comes within $r$ of $\tilde{p}$, and whose vertices are therefore within $R+2 d+r$ of $\tilde{p}$. As there are only finitely many such vertices, there are only finitely many such simplices $\sigma_{\omega}$ in the support of $z_{R, \infty}$.

Claim A.5 The chain $z_{R, \infty}$ is a locally finite cycle.

Proof We have just argued that $z_{R, \infty}$ is locally finite. The proof that $z_{R, \infty}$ is a cycle is nearly the same as the proof of [4, pages $115-116$, Claim (ii)], except that sums/unions over $\Gamma$ are replaced by sums/unions over $\hat{\Gamma}$, and conditions of the form " $u_{i} \in \gamma_{i}(D)$ " are replaced by " $u_{i} \in D(i)$ " for appropriately chosen pieces $D(i)$.

For $k \in \mathbb{N}$, let $\epsilon(k)$ be the smallest injectivity radius at any point in a piece of depth at most $k$, that is,

$$
\epsilon(k)=\inf \left\{\operatorname{inj}(V, x) \mid x \in P_{0} \text { or } x \in \bigcup\left\{P_{j, l} \mid j \in\{1, \ldots, m\}, l \leq k\right\}\right\} .
$$

It is not hard to see that as $k \rightarrow \infty$, the quantity $\epsilon(k)$ tends to zero. In particular, there is a constant $C$ so that if $k>C$, then $\epsilon(k)<\mu$.

Claim A.6 Let $R>0$, and let $C$ be such that $\epsilon\left(k^{\prime}\right)<\mu$ for all $k^{\prime}>C$. If $k>$ $R+C+2 d+1$, then $z_{R, k}$ is a relative cycle in $\left(V, V_{(0, \mu]}\right)$.

Proof Suppose an $(n-1)$-dimensional simplex $\sigma$ is in the support of $\partial z_{R, k}$. By Claim A.5, $\sigma$ must also be in the support of $\partial\left(z_{R, \infty}-z_{R, k}\right)$, so any lift $\tilde{\sigma}$ of $\sigma$ to $\mathbb{H}^{n}$ must have a vertex mapped to $\hat{\Gamma} \backslash \hat{\Gamma}_{k}$. The diameter of the image of $\tilde{\sigma}$ is at most $R+2 d$. Thus the vertices lie in $\hat{\Gamma} \backslash \widehat{\Gamma}_{k^{\prime}}$ for $k^{\prime}=k-\lceil R+2 d\rceil>C$, and no part of the image of $\tilde{\sigma}$ lies in a translate of the "fat piece" $D_{0}$. Since $\epsilon\left(k^{\prime}\right)<\mu, \sigma$ has image in the part of $V$ with injectivity radius less than $\mu$. 
Claim A.7 If $R>2 d$ then $a_{R}^{+}(\omega) \cdot a_{R}^{-}(\omega)=0$ for all $\omega \in \Omega$.

Proof This statement (and its proof) are identical to [4, pages 116-117, Claim (iii)].

Claim A.8 There is a quantity $\delta(R)>0$ so that $\lim _{R \rightarrow \infty} \delta(R)=0$ and so that the simplices in the support of $z_{R, \infty}$ all have volume at least $v_{n}-\delta(R)$.

Proof This statement is a slight rephrasing of [4, page 117, Claim (iv)]. The proof is the same.

Claim A.9 If $R>2 d$ and $a_{R}(\omega) \neq 0$, then $a_{R}(\omega) \operatorname{algvol}\left(\sigma_{\omega}\right)>0$.

Proof See [4, page 117, Claim (v)]. Claim A.7 is used here.

Claim A.10 Suppose $R>2 d+C$ and $k>2 R+1$, where $C$ is the constant from Claim A.6. The classes $\left[z_{R, \infty}\right] \in H_{n}^{l f}(V ; \mathbb{R})$ and $\left[z_{R, k}\right] \in H_{n}\left(V, V_{(0, \mu]} ; \mathbb{R}\right)$ are nontrivial.

Proof See [4, page 117, Claim (vi)]. The point here is just that some element of $\mathcal{S}(R)$ is a tuple of interior points of pieces corresponding to some tuple $\omega$, so that there is an open (and therefore positive measure) neighborhood of tuples of points all corresponding to the same tuple $\omega$.

We now complete the proof of the Proposition. Suppose $R>2 d+C$ and $k=\lceil 2 R\rceil+2$. It follows from Claim A.10 that there is some $\lambda>0$ so that $\lambda\left[z_{\infty, R}\right]$ is the fundamental class in the locally finite homology group $H_{n}^{l f}(V ; \mathbb{R})$ and some $\lambda^{\prime}$ so $\lambda^{\prime}\left[z_{k, R}\right]$ is the fundamental class in $H_{n}\left(V, V_{(0, \epsilon]} ; \mathbb{R}\right)$. In fact we must have $\lambda^{\prime}=\lambda$, since both $\lambda\left[z_{\infty, R}\right]$ and $\lambda^{\prime}\left[z_{k, R}\right]$ restrict to the orientation cycle at $x_{0}$, and the set of simplices in the supports of $z_{k, R}$ and $z_{\infty, R}$ which intersect a small neighborhood of $x_{0}$ is the same.

It follows from Claim A.9 that $\operatorname{algvol}\left(z_{k, R}\right) \leq \operatorname{algvol}\left(z_{\infty, R}\right)$. Moreover, an easy argument shows that if $c$ is a locally finite fundamental cycle for $V$, then $\operatorname{Vol}(V)=$ $\operatorname{algvol}(c)$. We therefore have

$$
\begin{aligned}
\operatorname{Vol}(V) & \geq \operatorname{algvol}\left(\lambda z_{k, R}\right) \\
& =\sum_{\omega \in \Omega_{k}} \lambda a_{R}(\omega) \operatorname{algvol}\left(\sigma_{\omega}\right) \\
& =\sum_{\omega \in \Omega_{k}} \lambda\left|a_{R}(\omega)\right| \cdot\left|\operatorname{algvol}\left(\sigma_{\omega}\right)\right| \\
& \geq\left(v_{n}-\delta(R)\right) \sum_{\omega \in \Omega_{k}} \lambda\left|a_{R}(\omega)\right| \\
& =\left(v_{n}-\delta(R)\right)\left\|\lambda z_{k, R}\right\|_{1} \geq\left(v_{n}-\delta(R)\right)\|M, \partial M\|,
\end{aligned}
$$


since $\lambda z_{k, R}$ gives a representative for the fundamental class of $(M, \partial M)$ via the homotopy equivalence of pairs $\left(V, V_{(0, \mu]}\right) \rightarrow(M, \partial M)$. Letting $R$ tend to infinity, Claim A.8 says that $\delta(R)$ tends to zero, so we have

$$
\operatorname{Vol}(V) \geq v_{n}\|M, \partial M\|,
$$

and the proposition is proved.

Lemma A.3 and Proposition A.4 together immediately imply Theorem A.1.

\section{Appendix B (Anti)Symmetrization of chains}

In this section we prove that the chain map $S$ defined in Section 2.2 is chain homotopic to the identity.

We let $\Delta^{n}$ denote the standard $n$-simplex, and begin by defining a "coning" operator for all $n$ and $k$

$$
c_{n}: C_{k}\left(\Delta^{n} ; \mathbb{R}\right) \rightarrow C_{k+1}\left(\Delta^{n} ; \mathbb{R}\right) .
$$

We define $c_{n}$ on a singular $k$-simplex $\psi: \Delta^{k} \rightarrow \Delta^{n}$ and then extend by linearity. Let $\iota: \Delta^{k} \rightarrow \Delta^{k+1}$ be the affine map taking each vertex $e_{i}$ of $\Delta^{k}$ to the vertex $e_{i+1}$ of $\Delta^{k+1}$; the vertex $e_{0}$ is the only one missed by $\iota$. Each point in $\Delta^{k+1}$ is uniquely expressible convex combination of some point in $\iota\left(\Delta^{k}\right)$ with $e_{0}$. Let $m_{n}$ be the barycenter of $\Delta^{n}$, and define

$$
c_{n}(\psi)\left(t e_{0}+(1-t) \iota(x)\right)=t m_{n}+(1-t) \psi(x) .
$$

In words, $c_{n}(\psi)$ is $\psi$ coned to the barycenter $m_{n}$. The key fact about $c_{n}$ is that for any chain $a \in C_{k}\left(\Delta^{n} ; \mathbb{R}\right)$, we have

$$
\partial c_{n}(a)=a-c_{n}(\partial a) .
$$

(In other words, $c_{n}$ is a chain contraction of $C_{*}\left(\Delta^{n} ; \mathbb{R}\right)$.)

We use the coning operator to inductively define an element $\mu_{n} \in C_{n+1}\left(\Delta^{n} ; \mathbb{R}\right)$, and, for any space $Y$, a map

$$
P_{n}^{(Y)}: C_{n}(Y ; \mathbb{R}) \rightarrow C_{n+1}(Y ; \mathbb{R}) .
$$

As in the definition of symmetrization, we abuse notation by using $q \in S_{n+1}$ to denote the unique affine map from $\Delta^{n}$ to itself which is equal to $q$ when restricted to the 
vertices. We start with $\mu_{0}=0$, and $P_{0}=0$ for all $Y$. We may assume $P_{n-1}^{\left(\Delta^{n}\right)}$ has been defined, and set

$$
\mu_{n}=\left(\frac{1}{\left|S_{n+1}\right|} \sum_{q \in S_{n+1}} \operatorname{sign}(q) c_{n}(q)\right)-c_{n}\left(\mathbf{1}_{\Delta^{n}}\right)-c_{n}\left(P_{n-1}^{\left(\Delta^{n}\right)}\left(\partial \mathbf{1}_{\Delta^{n}}\right)\right) .
$$

For any space $Y$, we define $P_{n}: C_{n}(Y ; \mathbb{R}) \rightarrow C_{n+1}(Y ; \mathbb{R})$ on simplices, and then extend linearly; for $\phi: \Delta^{n} \rightarrow Y$ a singular $n$-simplex, define

$$
P_{n}^{(Y)}(\phi)=\phi_{\sharp}\left(\mu_{n}\right) \text {. }
$$

The maps $P_{n}^{(\cdot)}$ commute with continuous maps:

Lemma B.1 If $f: X \rightarrow Y$ is any continuous map, and $n$ is an integer, then $f_{\sharp} \circ P_{n}^{(X)}=$ $P_{n}^{(Y)} \circ f_{\sharp}$.

Proof Since all the maps involved are linear, it suffices to verify this for a single singular simplex $\phi: \Delta^{n} \rightarrow X$. We have

$$
\left(f_{\sharp} \circ P_{n}^{(X)}\right)(\phi)=f_{\sharp} \phi_{\sharp}\left(\mu_{n}\right)=(f \circ \phi)_{\sharp}\left(\mu_{n}\right)=P_{n}^{(Y)}(f \circ \phi)=\left(P_{n}^{(Y)} \circ f_{\sharp}\right)(\phi) .
$$

Lemma B.2 $P_{*}^{(X)}$ as defined in equation (12) is a chain homotopy between $S$ and $\mathbf{1}_{C_{*}(X ; \mathbb{R})}$.

Proof In this proof we'll write $P_{k}^{(X)}$ as $P_{k}$.

It suffices to show that, for each $n$ and each singular $n$-simplex $\phi$,

$$
\partial P_{n}(\phi)=S(\phi)-\phi-P_{n-1}(\partial \phi) .
$$

For $n=0$, this is immediate, so we suppose that $n>0$ and argue inductively. We compute from (12) and (10),

$$
\begin{aligned}
\partial P_{n}(\phi)=\phi_{\sharp}\left(\frac{1}{\left|S_{n+1}\right|} \sum_{q \in S_{n+1}} \operatorname{sign}(q)\left(q-c_{n}(\partial q)\right)\right. \\
\left.\quad-\mathbf{1}_{\Delta^{n}}+c_{n}\left(\partial \mathbf{1}_{\Delta^{n}}\right)-P_{n-1}\left(\partial \mathbf{1}_{\Delta^{n}}\right)+c_{n} \partial\left(P_{n-1}^{\left(\Delta^{n}\right)}\left(\partial \mathbf{1}_{\Delta^{n}}\right)\right)\right)
\end{aligned}
$$

By induction on $n$ (and the fact that $\partial^{2}=0$ ) we have

$$
\partial P_{n-1}^{\left(\Delta^{n}\right)}\left(\partial \mathbf{1}_{\Delta^{n}}\right)=S\left(\partial \mathbf{1}_{\Delta^{n}}\right)-\partial \mathbf{1}_{\Delta^{n}},
$$


so we can rewrite

$$
\begin{aligned}
\partial P_{n}(\phi)=\phi_{\sharp}\left(\frac{1}{\left|S_{n+1}\right|} \sum_{q \in S_{n+1}} \operatorname{sign}(q)\left(q-c_{n}(\partial q)\right)\right. \\
\left.\quad-\mathbf{1}_{\Delta^{n}}+c_{n}\left(\partial \mathbf{1}_{\Delta^{n}}\right)-P_{n-1}^{\left(\Delta^{n}\right)}\left(\partial \mathbf{1}_{\Delta^{n}}\right)+c_{n}\left(S\left(\partial \mathbf{1}_{\Delta^{n}}\right)-\partial \mathbf{1}_{\Delta^{n}}\right)\right)
\end{aligned}
$$

The last two terms exactly cancel because $c_{n}$ and $\partial$ are both linear, so

$$
\frac{1}{\left|S_{n+1}\right|} \sum_{q \in S_{n+1}} \operatorname{sign}(q) c_{n}(\partial q)=c_{n} \partial\left(\frac{1}{\left|S_{n+1}\right|} \sum_{q \in S_{n+1}} \operatorname{sign}(q) q\right)=c_{n} \partial S\left(\mathbf{1}_{\Delta^{n}}\right)
$$

and, since $S$ is a chain map, $c_{n}\left(\partial S\left(\mathbf{1}_{\Delta^{n}}\right)\right)=c_{n}\left(S\left(\partial \mathbf{1}_{\Delta^{n}}\right)\right)$. We therefore have

$$
\partial P_{n}(\phi)=\phi_{\sharp}\left(S\left(\mathbf{1}_{\Delta^{n}}\right)-\mathbf{1}_{\Delta^{n}}-P_{n-1}^{\left(\Delta^{n}\right)}\left(\partial \mathbf{1}_{\Delta^{n}}\right)\right) .
$$

We now invoke Lemma B.1, which implies that

$$
\phi_{\sharp}\left(P_{n-1}^{\left(\Delta^{n}\right)}\left(\partial \mathbf{1}_{\Delta^{n}}\right)\right)=P_{n-1}(\partial \phi),
$$

and so

$$
\partial P_{n}(\phi)=S(\phi)-\phi-P_{n-1}(\partial \phi)
$$

\section{Acknowledgments}

We thank Danny Calegari, Mike Davis, Bill Thurston, Clara Löh, and Shmuel Weinberger for useful conversations. We also thank Alexander Nabutovsky for pointing out to us that our work applies to the homology spheres of Ratcliffe and Tschantz.

Jason Manning did part of this work during visits to the Caltech mathematics department and the Cornell mathematics department, and thanks Caltech and Cornell for their hospitality.

Koji Fujiwara is supported in part by a Grant-in-Aid for Scientific Research (No. 19340013). Jason Manning is supported in part by NSF grant DMS-0804369. 


\section{References}

[1] M T Anderson, Dehn filling and Einstein metrics in higher dimensions, J. Differential Geom. 73 (2006) 219-261 MR2225518

[2] L Babai, On Lovász' lattice reduction and the nearest lattice point problem, Combinatorica 6 (1986) 1-13 MR856638

[3] I Belegradek, Aspherical manifolds, relative hyperbolicity, simplicial volume and assembly maps, Algebr. Geom. Topol. 6 (2006) 1341-1354 MR2253450

[4] R Benedetti, C Petronio, Lectures on hyperbolic geometry, Universitext, Springer, Berlin (1992) MR1219310

[5] S A Bleiler, C D Hodgson, Spherical space forms and Dehn filling, Topology 35 (1996) 809-833 MR1396779

[6] S Francaviglia, Hyperbolic volume of representations of fundamental groups of cusped 3-manifolds, Int. Math. Res. Not. (2004) 425-459 MR2040346

[7] R Frigerio, C Pagliantini, The simplicial volume of hyperbolic manifolds with geodesic boundary, Algebr. Geom. Topol. 10 (2010) 979-1001 MR2629772

[8] K Fujiwara, J F Manning, CAT(0) and CAT(-1) fillings of hyperbolic manifolds, J. Differential Geom. 85 (2010) 229-269 MR2732977

[9] D Futer, E Kalfagianni, J S Purcell, Dehn filling, volume, and the Jones polynomial, J. Differential Geom. 78 (2008) 429-464 MR2396249

[10] M Gromov, Manifolds of negative curvature, J. Differential Geom. 13 (1978) 223-230 MR540941

[11] M Gromov, Volume and bounded cohomology, Inst. Hautes Études Sci. Publ. Math. (1982) 5-99 (1983) MR686042

[12] M Gromov, Hyperbolic groups, from: "Essays in group theory", Math. Sci. Res. Inst. Publ. 8, Springer, New York (1987) 75-263 MR919829

[13] M Gromov, W Thurston, Pinching constants for hyperbolic manifolds, Invent. Math. 89 (1987) 1-12 MR892185

[14] D Groves, J F Manning, Dehn filling in relatively hyperbolic groups, Israel J. Math. 168 (2008) 317-429 MR2448064

[15] S K Hansen, Measure homology, Math. Scand. 83 (1998) 205-219 MR1673914

[16] C D Hodgson, S P Kerckhoff, Universal bounds for hyperbolic Dehn surgery, Ann. of Math. (2) 162 (2005) 367-421 MR2178964

[17] G C Hruska, B Kleiner, Hadamard spaces with isolated flats, Geom. Topol. 9 (2005) 1501-1538 MR2175151 With an appendix by the authors and M Hindawi

[18] D Ivanšić, Hyperbolic structure on a complement of tori in the 4-sphere, Adv. Geom. 4 (2004) 119-139 MR2155369 
[19] A K Lenstra, H W Lenstra, Jr, L Lovász, Factoring polynomials with rational coefficients, Math. Ann. 261 (1982) 515-534 MR682664

[20] C Löh, Measure homology and singular homology are isometrically isomorphic, Math. Z. 253 (2006) 197-218 MR2206643

[21] I Mineyev, Bounded cohomology characterizes hyperbolic groups, Q. J. Math. 53 (2002) 59-73 MR1887670

[22] I Mineyev, A Yaman, Relative hyperbolicity and bounded cohomology, preprint Available at http://www.math.uiuc.edu/ mineyev/math/art/rel-hyp.pdf

[23] L Mosher, M Sageev, Nonmanifold hyperbolic groups of high cohomological dimension, preprint (1997) Available at http://andromeda.rutgers.edu/ mosher/ HighDHyp.ps.gz

[24] A Nabutovsky, S Weinberger, Variational problems for Riemannian functionals and arithmetic groups, Inst. Hautes Études Sci. Publ. Math. (2000) 5-62 (2001) MR1839486

[25] W D Neumann, D Zagier, Volumes of hyperbolic three-manifolds, Topology 24 (1985) 307-332 MR815482

[26] J G Ratcliffe, Foundations of hyperbolic manifolds, Graduate Texts in Mathematics 149, Springer, New York (1994) MR1299730

[27] J G Ratcliffe, S T Tschantz, Some examples of aspherical 4-manifolds that are homology 4-spheres, Topology 44 (2005) 341-350 MR2114711

[28] V Schroeder, A cusp closing theorem, Proc. Amer. Math. Soc. 106 (1989) 797-802 MR957267

[29] W P Thurston, Geometry and Topology of Three-Manifolds, lecture notes, Princeton (1980) Available at http://www.msri.org/publications/books/gt3m/

[30] H C Wang, Topics on totally discontinuous groups, from: "Symmetric spaces (Short Courses, Washington Univ., St. Louis, Mo., 1969-1970)”, Dekker, New York (1972) 459-487. Pure and Appl. Math., Vol. 8 MR0414787

[31] T Yamaguchi, Simplicial volumes of Alexandrov spaces, Kyushu J. Math. 51 (1997) 273-296 MR1470157

[32] A Zastrow, On the (non)-coincidence of Milnor-Thurston homology theory with singular homology theory, Pacific J. Math. 186 (1998) 369-396 MR1663826

Graduate School of Information Sciences, Tohoku University

Sendai, 980-8579, Japan

Department of Mathematics, University at Buffalo, SUNY

Buffalo NY 14260, USA

fujiwara@math.is.tohoku.ac.jp, j399m@buffalo.edu

Received: 2 February 2011 